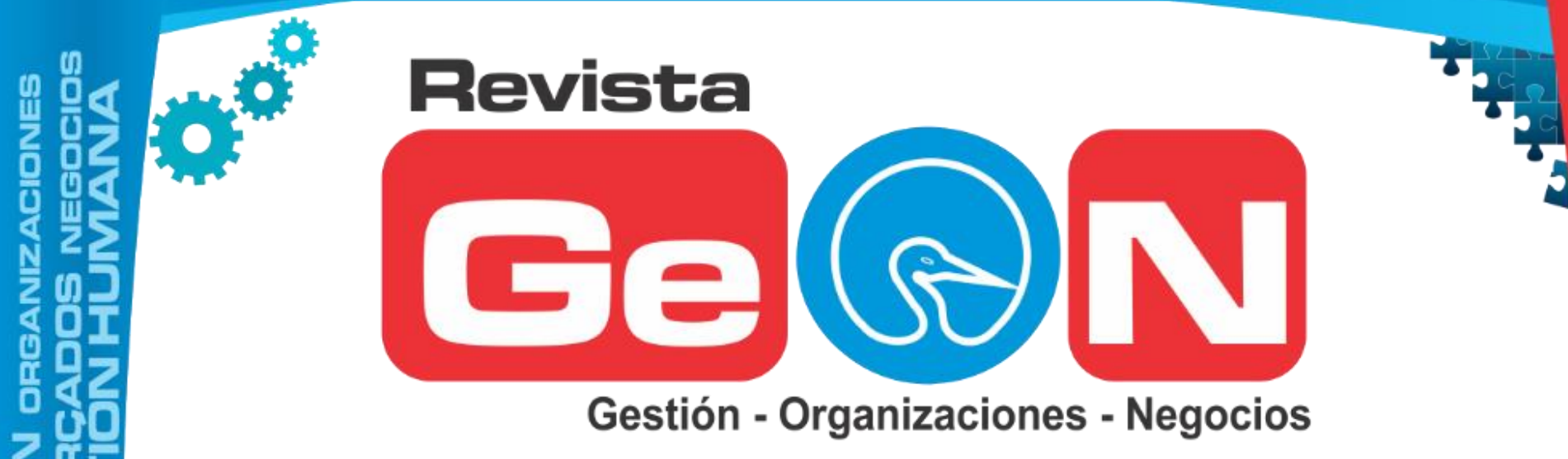

2 it ISEN EC4E - 3910 Volumén E No 1 Enero a Junio E015

Revista Electrónica de la Facultad de Ciencias Económicas de la Universidad de los Llanos

는 Revista Electronica de la FaculaVICENCIO- COLOMBIA

$4 \frac{4}{2}$

04

72

21

- 2

단

(i)

ii III

(ㄴ)

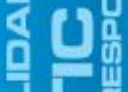

IiI
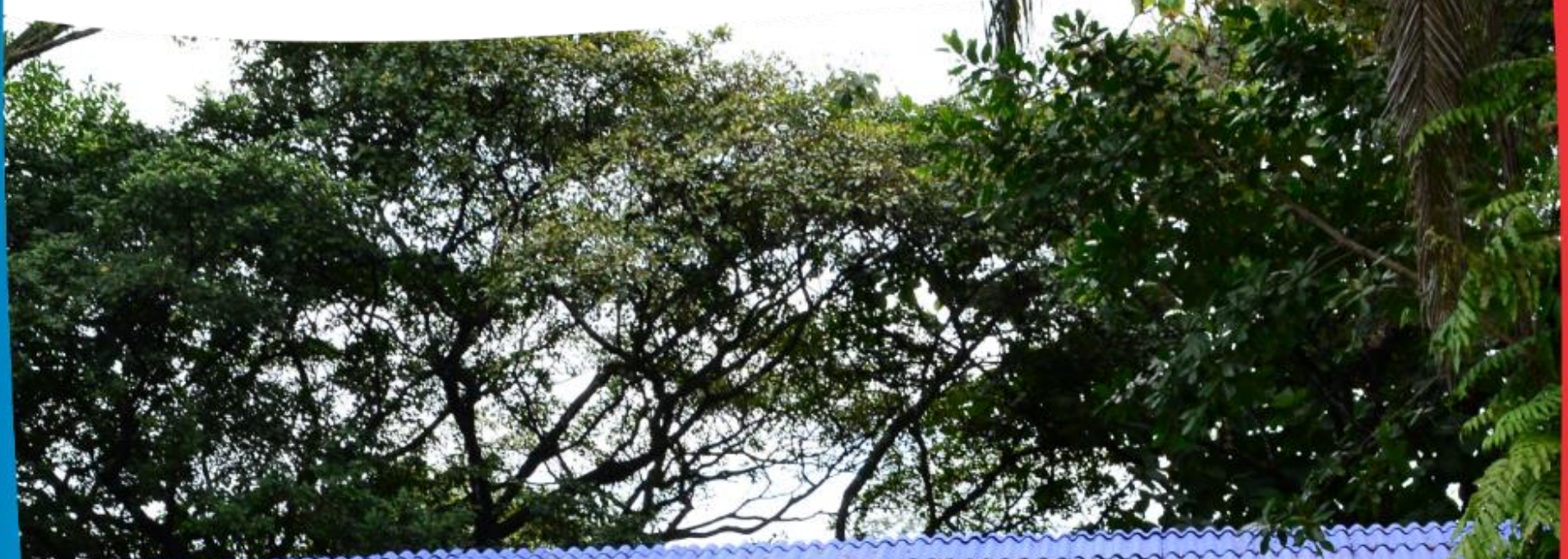

III) in 0

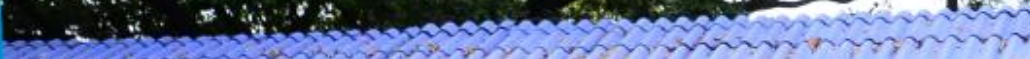

0

iii

î. II

40

82

T

80

更 II

3

,

2

20

QN

42

04

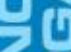

5 iा

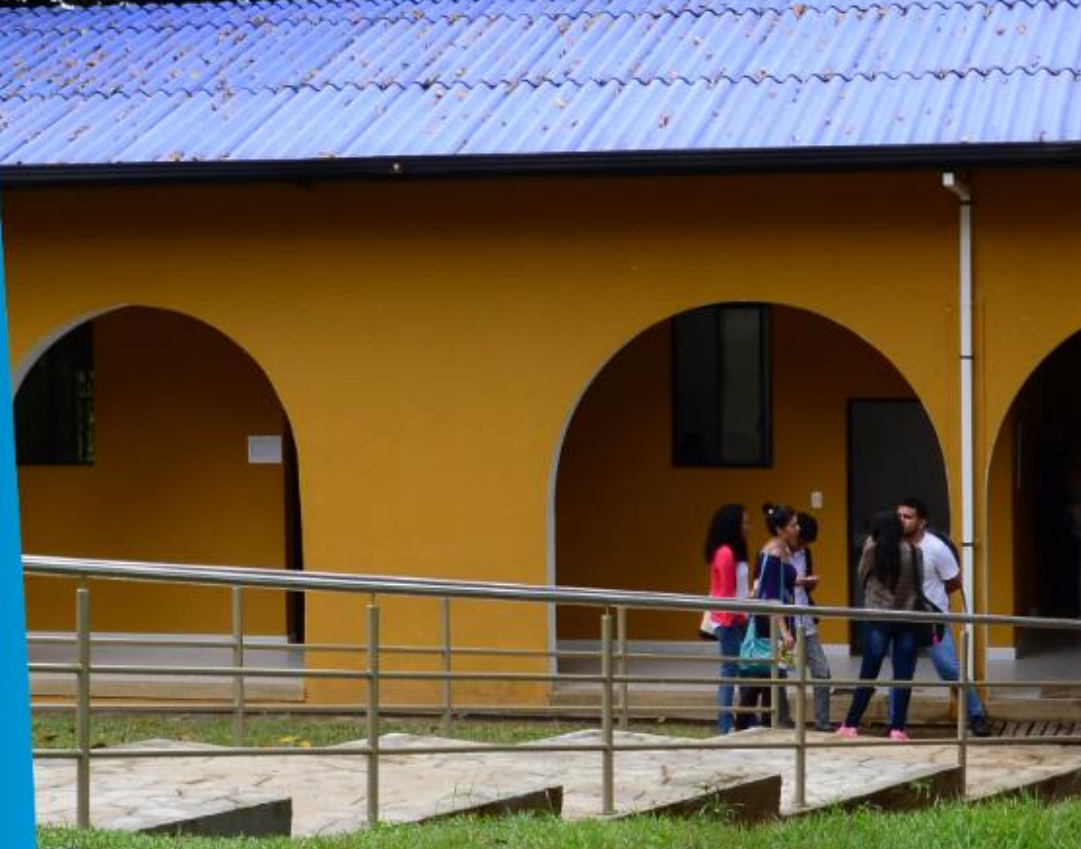

-

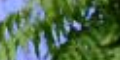




\section{Proceso de vinculación del Recurso Humano a contratar por el Centro de Innovación Educativo Regional Oriente -CIER Oriente-, en la ciudad de Villavicencio - por Viviana Pérez}

Leidy Viviana Pérez Ibarra

Administradora de Empresas

Viviana.lvpi@gmail.com

@ viviana_lvpi

\section{Resumen}

El objetivo del presente trabajo de grado es desarrollar una propuesta administrativa que oriente un proceso de vinculación de recurso humano a contratar por el Centro en Innovación Educativa Regional Oriente -CIER Oriente-, en la ciudad de Villavicencio. La propuesta se enmarcó dentro de teorías administrativas que direccionaron la elaboración de los subprocesos tales como el reclutamiento, selección y contratación de recurso humano, necesarios para el desarrollo de la vinculación del personal, el cual se ha caracterizado por la recopilación de información, actualización y mecanismos de mejora.

El desarrollo de la propuesta involucra la participación de empleados del CIER Oriente y entidades reguladoras como es la Universidad de los Llanos en Villavicencio y el Ministerio de Educación Nacional.

\section{Palabras clave}

Centro de Innovación Educativo Regional (CIER Oriente); Ministerio de Educación Nacional (MEN); Recurso humano; Reclutamiento; Selección; Contratación.

\section{Abstract}

The aim of this work is to develop a management degree proposal that guides a process of linking human resources to hire by the Center on Education Innovation Regional East- East -CIER in the city of Villavicencio. The proposal is framed within administrative theories processing threads such as recruitment, selection and hiring of human resources necessary for the development of linkage staff, which has been characterized by information gathering , updating and improvement mechanisms .

The development of the proposal involves the participation of employees in the CIER East and regulatory bodies such as the Universidad de los Llanos in Villavicencio and the Ministry of Education.

\section{Keywords}

East- Regional Educational Innovation Center; National Education Ministry; Human Resources; Recruiting; Selection; Hiring.

\section{Introducción}

Colombia, en el sector de educación por medio del Ministerio de Educación, desde 1990 ha adelantado estrategias de dotación de infraestructura, tecnología, aplicaciones educativas y formación del recurso humano docente.

El Ministerio de Educación Nacional, cumpliendo con las políticas presentadas en el plan sectorial 2010-2014, consolidó el Sistema Nacional de Innovación Educativa con Uso de TIC para contribuir con el fortalecimiento de la calidad de la educación, desde la cual se apoye la formación del capital humano que demanda el país para atender los múltiples desafíos que ha traído el nuevo milenio.

Una de las estrategias tomadas a través de su Oficina de Innovación Educativa, fue lanzar en junio del 2012 oficialmente una convocatoria para la selección de las alianzas encargadas de la operación, administración y gestión desde Cinco Centros de Innovación Educativa Regional (CIER), en la página de Colombia aprende Ministerio de Educación Nacional.

Cada uno de los cinco centros son áreas geográficas integradas por departamentos, en las cuáles se ha dividido el país para efectos de esta 
convocatoria que tiene como objeto garantizar la atención y cobertura nacional del proyecto (Mineducación, 2014).

Actualmente el proyecto está en desarrollo y con el fin de garantizar eficiencia, optimizar productividad y aumentar la competitividad, el grupo base visualizó la necesidad de desarrollar un proceso de vinculación de personal que se ajustara a los requerimientos del Ministerio de Educación Nacional y a las necesidades propias del proyecto determinadas por el director y los coordinadores de las unidades, en cuanto al recurso humano total requerido a contratar para el CIER Oriente.

Partiendo de que el propósito es diseñar una propuesta para estructurar el proceso de vinculación del personal requerido por el Centro de Innovación Educativo Regional CIER Oriente, el desarrollo de la propuesta tendrá como base la administración de recursos humanos.

\section{Desarrollo}

El análisis situacional es un método que permite analizar dificultades, fallas, oportunidades y riesgos, para definirlos, clasificarlos, desglosarlos, jerarquizarlos y ponderarlos, permitiendo así actuar eficientemente con base en criterios y/o planes establecidos (Hanel y Hanel, 2004).

El propósito fundamental del diagnóstico fue conocer la situación actual del -CIER Oriente-, detectando las necesidades básicamente en recurso humano.

A continuación se encuentra la información recolectada por medio de este instrumento, que a través de preguntas semiestructuradas se aplicó al Director del CIER Oriente ${ }^{5}$.

La recolección de información a través de la entrevista permite explicar y comprender las interrelaciones entre variables que están

\footnotetext{
${ }^{5}$ Luis Facundo Maldonado Granados, actual Director del CIER Oriente, con Ph.D. en Sistemas para Educación de la Florida State University, Magister en Psicopedagogía y en Investigación y Análisis Curricular de la Universidad Pedagógica Nacional; Especialista en Administración Pública de la Escuela Superior de Administración Pública - ESAP, entre otros estudios.
}

determinando una situación real y por lo tanto permiten encontrar acciones de mejora.

El MEN propone para el logro de propósitos incluir cuatro frentes de trabajo:

1. Infraestructura. Integra la dotación física y tecnológica; inicia con recursos de contrapartida por la alcaldía de Villavicencio, que construyó un edificio destinado al laboratorio multimedia y a una sala de producción de contenidos digitales. De igual forma la Universidad de los Llanos realiza la adaptación de cuatro aulas para formación, dos espacios para investigación $\mathrm{y}$ producción, y una para operación del CIER.

2. Unidad de producción de contenidos digitales. Diseña, desarrolla e implementa en el portal Colombia Aprende, 6060 recursos digitales (DR) en 202 objetos de aprendizaje (LO) distribuidos en las áreas de ciencias, matemáticas y lenguaje para los grados $4^{\circ}$ y $5^{\circ}$ de primaria.

3. Formación docente. Contribuye mediante la formación de 3000 docentes de la región oriental de Colombia, y al fortalecimiento de uso educativo de las TIC.

4. Unidad administrativa. Es un componente que se desarrolla desde el CIER Oriente, con el fin de ser sostenible en el tiempo. A través de esta unidad se pretende desarrollar diferentes proyectos y apoyar procesos internos del CIER Oriente, todo mediante la dirección y asistencia de actividades.

Tabla 1

Personal faltante en el CIER Oriente.

\begin{tabular}{lc}
\hline \multicolumn{1}{c}{ Cargo } & CIER \\
\hline Soporte Técnico en infraestructura & 1 \\
\hline Soporte técnico audio visual & 1 \\
\hline Administrador de aulas para formación & 1 \\
\hline Formador de Formadores & 16 \\
\hline Coordinador Unidad de Contenidos & 1 \\
\hline Desarrollador Multimedia & 2 \\
\hline Camarógrafo & 1 \\
\hline Productor/Editor & 1 \\
\hline Desarrollador de contenidos & 3 \\
\hline Diseñador Instruccional & 3 \\
\hline Experto Pedagogo para apoyo temático & 3 \\
\hline Programador & \\
\hline Fuente: Elaboración propia con base a información del anexo técnico \\
Centros de Innovación Educativa Regional -CIER-. Proyecto “ICT \\
EDUCATION CAPABILITIES BUILDING”. Item 5. Recurso Humano. \\
Ministerio de Educación.
\end{tabular}


Proceso de vinculación del Recurso Humano a contratar por el Centro de Innovación Educativo Regional Oriente -CIER Oriente-, en la ciudad de Villavicencio - por Viviana Pérez

Según el anexo técnico Centros De Innovación Educativa Regional - CIER Proyecto "ICT EDUCATION CAPABILITIES BUILDING", ítem 5, Recurso Humano, se realiza un re-ajuste a la asignación del personal requiriendo contratar en total 53 cargos en diferentes áreas, de los cuales cuenta con contrato siete cargos: Director, Asistente general, Experto E-Learning, Experto en infraestructura, Experto en contenidos, Experto en formación/pedagogía y Coordinador Unidad de contenidos, lo que quiere decir que en total son 46 cargos los faltantes.

Al conocer la necesidad se diseña un análisis DOFA con base en un análisis del ambiente interno y externo. El análisis interno determina las fortalezas y debilidades del proyecto, el análisis externo determina las oportunidades $\mathrm{y}$ amenazas del proyecto.

Con un enfoque especial en el área de recurso humano en el -CIER Oriente-, se desarrollaron cuatro tipos de estrategias: (FO) fortalezasoportunidades, (DO) debilidades-oportunidades, (FA) fortalezas-amenazas y (DA) debilidadesamenazas. Dentro de estas se encuentran:

FO: Sensibilizar al área de jurídica de la Universidad de los Llanos sobre la importancia del proceso de contratación que estará a su cargo, siendo este un proyecto a nivel nacional e internacional.

DO: Diseñar el proceso de vinculación que incluye reclutamiento, selección y contratación del recurso humano, orientadas a identificar las competencias conductistas y funcionalistas, que garanticen perfiles laborales enfocados a los objetivos del proyecto.

FA: Apoyar el proceso de reclutamiento no solo con el banco de hojas de vida del Ministerio, sino a través de una promoción interna y externa de vacantes.

DA: Desarrollar un sistema de selección de personal que enfoque los perfiles requeridos (pruebas de conocimiento).

De acuerdo a estas estrategias se inicia el proceso de reclutamiento, debe atraer suficiente cantidad de candidatos para abastecer de modo adecuado el proceso de selección (Chiavenato, 2001).
Dicha planeación del reclutamiento de recurso humano en el CIER Oriente exige una sistematización que cuente con tres fases que se mencionan en la referida obra de Chiavenato (2001), iniciando con una investigación interna, investigación externa y por ultimo unas técnicas de reclutamiento.

La investigación interna en el CIER Oriente es el proceso de decisión respecto a las necesidades de personal, perfiles requeridos $\mathrm{y}$ aptitudes, se resume en lo que requiere la organización.

La descripción de los cargos faltantes se realiza a través de un formato de descripción de cargos de acuerdo al consolidado de la tabla 2 . Se toman además como guía los formatos para la gestión de salarios e incentivos y se realizan las adaptaciones pertinentes y necesarias para los propios requisitos.

De este modo, el formato está divido en IV partes: I. Identificación del cargo, II. Resumen del cargo, III Funciones del cargo, IV. Especificaciones del cargo.

Teniendo en cuenta que cada CIER deberá seleccionar y contratar el recurso humano adicional, se propone la adición de los siguientes cargos para el proyecto del CIER Oriente, para el área administrativa:

Tabla 2

Cargos adicionales.

\begin{tabular}{lc}
\hline \multicolumn{1}{c}{ Cargo } & CIER \\
\hline Auxiliar contable & 1 \\
\hline $\begin{array}{l}\text { Coordinador interinstitucional y de } \\
\text { promoción }\end{array}$ & 1 \\
\hline
\end{tabular}

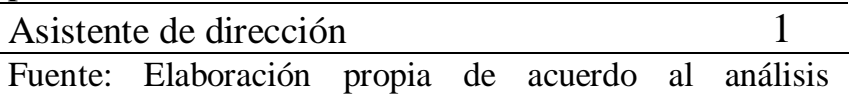
situacional.

De la investigación externa sobresalen dos aspectos importantes: la segmentación del mercado de recursos humanos y las fuentes de reclutamiento (Chiavenato, 2001).

La segmentación del mercado busca la división del personal en las áreas afines, con el fin de realizar una caracterización de los candidatos de acuerdo a la unidad en la cual este participando; por tal razón se acoplan las 
Proceso de vinculación del Recurso Humano a contratar por el Centro de Innovación Educativo Regional Oriente -CIER Oriente-, en la ciudad de Villavicencio - por Viviana Pérez

unidades con los diferentes frentes de trabajo, como se organiza en la siguiente tabla:

Tabla 3

Segmentación del recurso humano. UNIDAD DE INFRAESTRUCTURA

Soporte Técnico en infraestructura (UDF)

Soporte técnico audio visual (UDC)

UNIDAD DE FORMACIÓN DOCENTE

Administrador de aulas para formación

Formador de Formadores

UNIDAD DESARROLLO DE CONTENIDOS

Coordinador Unidad de Contenidos

Desarrollador Multimedia

Camarógrafo

Productor/Editor

Desarrollador de contenidos para la producción

Diseñador Instruccional

Experto Pedagogo para apoyo temático

Programador

Asistente de dirección

Auxiliar contable

Coordinador interinstitucional y de promoción

Fuente: Elaboración propia con base a información de los términos de referencia y el Anexo Técnico Centros de Innovación Educativa Regional - CIER. Proyecto "ICT Education Capabilities Building".

Una vez segmentado el recurso humano, el mercado de recurso humano presenta diversas fuentes que la empresa debe identificar $y$ localizar, con el propósito de atraer candidatos que suplan sus necesidades a través de múltiples técnicas de reclutamiento (Chiavenato, 2001).

Los tres medios de reclutamiento son interno, externo y mixto.

El interno se basa en la investigación para suplir la necesidad de recurso humano, a través de la reubicación. Éste puede ser por transferencia vertical, movimiento horizontal o diagonal. Es necesario que el reclutamiento tenga una interacción constante con las demás dependencias. El externo busca suplir la necesidad con candidatos que no pertenecen al proyecto y el mixto compone las dos técnicas, ya que al realizar el interno es necesario cubrir el cargo que deja el empleado anterior, a través del externo.
Los medios que se proponen son vistos como estrategias de transferencia o divulgación que buscan lograr un reclutamiento óptimo de recurso humano.

Reubicación del recurso humano. Este movimiento interno beneficia al CIER Oriente, generando seguridad, puesto que ya se conoce al candidato y aprovecha las inversiones de capacitación. Es necesario tener acceso a las pruebas de selección como entrevistas, resultados de los programas de capacitación y un análisis del cargo que está desempeñando.

Candidatos Unillanos. El reclutamiento externo se llevará a cabo inicialmente en la universidad de los Llanos para el personal contratado por prestación de servicios, teniendo en cuenta que los perfiles requeridos por el CIER Oriente tienen una carga laboral de tiempo completo. La información se montará en la página web de la universidad y se realizará una descripción total de los cargos y perfiles requeridos.

Candidatos contactados por los aliados del CIER Oriente. Ya que el CIER Oriente está integrado bajo una alianza por la Universidad de los Llanos, Biollanos Ingeniería Limitada, Universidad Nacional de Colombia - Instituto de Biotecnología y Latin Campus Corporate University (I3Net SAS), Sec. Edu. Villavicencio, Sec. Edu. Guainía, Sec. Edu. Guaviare, Sec. Edu. de Vaupés y Sec. Edu. Arauca, se solicitará a los aliados el apoyo en la postulación de candidatos.

Candidatos presentados por empleados del CIER Oriente: Con el fin de realizar un reclutamiento con alto rendimiento y bajo índice de tiempo, el CIER Oriente estimulará a los contratistas a presentar y recomendar candidatos que se ajusten a los perfiles requeridos.

Candidatos presentados por CIER de Colombia. Ya que son cinco Centros de Innovación Educativas Regionales -CIER, Se convocará a los Centros para establecer un apoyo en el reclutamiento de personal, teniendo en cuenta que cada uno está diferenciado en regiones.

Candidatos contactados por entidades educativas. Ya que el proyecto es liderado por la oficina de innovación del Ministerio de 
Educación Nacional, se solicitará un apoyo al Servicio de Apoyo Nacional -SENA, primeramente para divulgar las vacantes de forma física y tecnológica (página web) y seguidamente para acceder a la base de datos, con el fin de conocer y ratificar información.

Anuncios en medios de comunicación. Por último se utilizará medios de comunicación, como la prensa, radio y la página web del CIER Oriente, con el fin de divulgar la necesidad de contratación.

La selección de personal es escoger entre los candidatos reclutados los más adecuados, para ocupar cargos existentes en la empresa, tratando de mantener la eficiencia de la organización (Chiavenato, 2001).

La selección del personal requerido se desarrolla en tres etapas. Etapa 1. Preselección. Los candidatos atraídos por el reclutamiento mixto a través de las siete estrategias postuladas en el proceso de reclutamiento. Etapa 2. Técnicas de selección. El CIER Oriente utilizará diferentes técnicas, con el fin de que se escojan los candidatos más idóneos; bajo el instrumento de la entrevista semiestructurada estructurada en III fases, fase I. confirmación u obtención de datos personales, formación académica, experiencia laboral, experiencia profesional, evidencias, aspectos económicos del contrato, fase II. Prueba de conocimiento, fase III. Entrevista de competencias requeridas. Etapa 3. Selección. Para llevar a cabo una selección óptima es necesario realizar un análisis y control de resultados, con el fin de que la selección sea eficaz y eficiente teniendo en cuenta los resultados de la entrevista. A la entrevista general se le asigna un porcentaje de $30 \%$, prueba de conocimiento $40 \%$ y a la entrevista por competencias de $30 \%$, para un total de $100 \%$.

El diseño del proceso de contratación busca ser una herramienta que garantice una adecuada planeación de la actividad contractual, permitiendo identificar el paso a paso de las actividades para suplir la necesidad.

Una vez seleccionado el personal idóneo, es necesario precisar los órganos competentes para contratar con el fin de formalizar el ingreso de dicho personal al Centro de Innovación
Educativa Regional Oriente, a través del operador la Universidad de los Llanos. Dicha vinculación se realizará bajo un contrato de prestación de servicios, como se determinó en la entrevista a cada cargo.

Los documentos necesarios para la contratación son: requerimiento para contratación de personal, necesidad de contratación, estudio de necesidad de personal, propuesta por el profesional, requisitos establecidos lista de chequeo, suscripción minuta, resolución rectoral de supervisión, certificado de disponibilidad presupuestal, registro presupuestal, aprobación de póliza, notificación supervisor, acta de inicio.

\section{Conclusiones}

De la propuesta administrativa para el proceso de vinculación del recurso humano a contratar por el centro de innovación educativo regional oriente -CIER Oriente-, en la ciudad de Villavicencio, se desprenden una serie de conclusiones, que se clasificaron en cuatro principales, teniendo en cuenta que se estructuró cuatro objetivos específicos para el desarrollo, en el cual se organiza un paso a paso en los subprocesos necesarios para la vinculación del personal, como es el proceso de reclutamiento, proceso de selección y el proceso de contratación, todo de acuerdo a las necesidades reales del proyecto, que se determina en un enfoque en el área de recurso humano.

El análisis situacional permitió examinar a través del diseño de una matriz DOFA las exigencias del Ministerio de educación, y las opciones de mejora de acuerdo a las necesidades del proyecto.

El proceso de reclutamiento se desarrolló en tres fases, las cuales son un conjunto de técnicas $\mathrm{y}$ procedimientos orientados a atraer los candidatos necesarios potenciales calificados y capaces de ocupar cargos dentro de la organización a través de investigaciones internas, externas y técnicas de reclutamiento.

Ya que el mercado de recurso humano presenta diversas fuentes o técnicas que la propuesta debió identificar y localizar con un reclutamiento mixto, ya que incluye el interno 
que se basa en la investigación para suplir la necesidad de recurso humano, a través de la reubicación, transferencia vertical, movimiento horizontal o diagonal, el externo que busca suplir la necesidad con candidatos que no pertenecen al proyecto, y el mixto compone las dos técnicas. Los medios o fuentes de reclutamiento mixto son vistos como estrategias de transferencia o divulgación que buscan lograr un reclutamiento óptimo de recurso humano: reubicación del recurso humano del CIER Oriente, candidatos postulados de la Universidad de los Llanos, candidatos contactados por los aliados del CIER Oriente, candidatos presentados por los empleados del CIER Oriente, candidatos presentados por los diferentes CIER de Colombia, candidatos contactados por entidades educativas de Villavicencio y anuncios en diferentes medios de comunicación.

El proceso de selección de personal o recurso humano se encargó de la elección del individuo, escogiendo entre los candidatos reclutados los más adecuados para ocupar cargos existentes en el proyecto, a través de tres etapas. Con el supuesto de candidatos atraídos por el reclutamiento mixto se pretende realizar una lista del recurso humano postulado, y con los requerimientos estipulados en el proceso de reclutamiento se diseña una base de datos realizando un primer filtro de preselección.

Continuamente dentro de las técnicas de selección se elaboran unos formatos de entrevista (general y por competencias) y se incluye una prueba de conocimiento para cada uno de los cargos, lo cual se evalúa con un porcentaje en cada uno de los formatos, esto con el fin de tomar la mejor opción.

El proceso de contratación buscó ser una herramienta que garantizara una adecuada planeación de la actividad contractual, permitiendo identificar el paso a paso de las actividades para suplir la necesidad. Muestra el procedimiento de contratación, lo cual indica que inicia desde la realización de requerimientos, pasando por diferentes formatos, hasta la aprobación y firma del acta de inicio por parte del representante legal del proyecto que en este caso es el rector de la Universidad de los
Llanos. Este proceso determina la actividad, descripción y responsable de la misma, cuyo objeto es crear un mecanismo de control en el proceso, de acuerdo a los lineamientos de la dirección y normatividad de la oficina de jurídica de la Universidad de los Llanos.

\section{Referencias}

Colombia Aprende, La red del conocimiento (2014). "Términos de referencia definitivos convocatoria para: Selección de las alianzas encargadas de la operación, administración y gestión de los centros de innovación educativa regional", sitio web de Colombia Aprende, [en línea], disponible en: http://www.colombiaaprende.edu.co/html/produ ctos/1685/articles-302543_recurso_1.pdf [accesado el día 17 de marzo de 2015].

Chiavenato, Idalberto (2001). Administración de recursos humanos. $5^{\circ}$ Edición. Bogotá: McGrawHill.

Hanel, Jorge y Hanel, Martha (2004). Análisis Situacional. Módulo II. México. Trillas.

Ministerio de Educación Nacional (2014). "Términos de referencia", sitio web de Mineducación, [en línea], disponible en: http://www.mineducacion.gov.co/1621/articles306952_archivo_pdf_terminos_junio8.pdf [accesado el día 17 de marzo de 2015]. 